Correspondencia a: Tarciso Rusivel. ${ }^{1}$ Mauricio Soto ${ }^{1}$. Tamara Viscarra. Luis Lafuente. ${ }^{1}$

Mikael Agreda. ${ }^{2}$

Sarali Orellana. ${ }^{2}$

${ }^{1}$ Estudiantes de Medicina de la Universidad Técnica Privada Cosmos.

Centro de Investigación Medicina UNITEPC. CIMU Cochabamba - Bolivia

${ }^{2}$ Asesor, docente de Medicina de la Universidad Técnica Privada Cosmos. Cochabamba - Bolivia

sorellana_investigacion@ unitepc-mail.com magreda_investigacion@ unitepc-mail.com

Procedencia y arbitraje: no comisionado, sometido a arbitraje externo

Recibido para publicación: 30 de marzo de 2016

Aceptado para publicación: 6 de junio de 2016

Citar como:

$\mathrm{Re} \mathrm{Ci} \mathrm{Sa}$ UNI

2016; 1(2):10-14

\section{PREVALENCIA DE SÍFILIS EN GESTANTES VILLA PAGADOR 2015}

\section{PREVALENCE OF SIFILIS IN GESTANTES VILLA PAGADOR 2015}

\section{PREVALÊNCIA DE SIFILIS EM GRÁVIDA VILLA PAGADOR 2015}

\section{RESUMEN.}

La Sífilis Congénita es una enfermedad que va en incremento a pesar de los controles, los estímulos y de la educación sexual brindada a la población el objetivo es establecer la prevalencia de Sífilis en mujeres embarazadas que acudieron para hacer sus controles prenatales al Hospital de Villa Pagador. Se realizó un estudio tipo retrospectivo, descriptivo de corte transversal. El grupo poblacional consiste en mujeres embarazadas que hicieron su control laboratorial antes de las 20 semanas de gestación. Durante el 2015 se hicieron laboratorio a 972 mujeres, 7 resultaron positivo, 1\%. En el presente trabajo se encontró una prevalencia de sífilis materna en el hospital de Villa Pagador de 1\%, menor a la prevalencia global de sífilis materna de 4,3\% reportada para Bolivia en 1996.

Palabras claves: Prevalencia, Sífilis, Gestante.

\section{ABSTRACT.}

Congenital syphilis is a disease that is increasing despite the controls, stimuli and sex education provided to the population. The objective is to establish the prevalence of syphilis in pregnant women who came to do their prenatal check-ups at Villa Pagador Hospital . A retrospective, descriptive cross-sectional study was carried out. The population group consists of pregnant women who made their laboratory control before 20 weeks of gestation. During 2015, 972 women were laboratory, 7 were positive, 1\%. In the present study, a prevalence of maternal syphilis was found in the Villa Pagador hospital of $1 \%$, lower than the global prevalence of maternal syphilis of 4.3\% reported for Bolivia in 1996.

Keywords: Prevalence. Syphilis. Gestants

RESUMO. 
A sífilis congênita é uma doença que vem aumentando, apesar dos controles, estímulos e educação sexual fornecidos à população, com o objetivo de estabelecer a prevalência de sífilis em gestantes que realizaram o pré-natal no Hospital Villa Pagador. Foi realizado um estudo retrospectivo, descritivo e transversal. O grupo escolhido consiste em mulheres grávidas que fizeram o controle laboratorial antes das 20 semanas de gestação. Durante o ano de 2015, 972 mulheres foram aos seus controles laboratoriais, 7 foram positivas, $1 \%$. No presente estudo, foi encontrada uma prevalência de sífilis materna no hospital Villa Pagador de $1 \%$, inferior à prevalência global de sífilis materna de 4,3\% relatada para a Bolívia em 1996.

Palavras chave: Prevalência, sífilis, gravidez.

\section{INTRODUCCIÓN.}

La sífilis es una infección multisistémica provocada por la bacteria Treponema Pallidum. A pesar de que la sífilis materna aún representa un problema de Salud Pública en América Latina la distribución mundial afecta a 12 millones de personas cada año con alta morbilidad y mortalidad.

La prevención y control de la infección materna por el virus de inmunodeficiencia humana $(\mathrm{VIH})$, la vía de contagio más importante es la sexual, que deriva en distintas formas clínicas como son: sífilis primaria, sífilis secundaria, sífilis latente precoz y tardía y, sífilis terciaria. ${ }^{1}$

Los recién nacidos se pueden transmite desde una madre infectada durante todo el embarazo por vía transparentaría (vía más frecuente de transmisión en recién nacido), o a través del canal del parto, pudiendo derivar ambas en dos formas de presentación clínica: sífilis congénita precoz y tardía. ${ }^{2}$

La Sífilis Congénita es una enfermedad que va en incremento a pesar de los controles, los estímulos y de la educación sexual brindada a la población.

Las cifras son lamentables, es así como el $90 \%$ de los Recién Nacidos de madres infectadas con sífilis no tratada, tendrán sífilis congénita, e incluso muchos de ellos desarrollaran posteriormente la enfermedad hasta dos o tres meses después del contacto inicial; en este caso, las manifestaciones de la infección, pueden ser realmente limitantes para el desarrollo psicomotor del niño dando lugar a sordera, déficit neurológico, retraso del crecimiento y deformidades óseas. Un $34 \%$ de los niños afectados mueren antes de nacer, el $50 \%$ mueren después de nacer con o sin tratamiento, el $75 \%$ son asintomáticos y un $60 \%$ se logran diagnosticar después del año de vida ${ }^{1,2}$. La sífilis congénita se puede prevenir y tratar eficazmente in útero, siempre y cuando el diagnóstico se haga en forma oportuna. Para prevenir la sífilis congénita, en Bolivia se ha determinado la pesquisa de la enfermedad en mujeres gestantes mediante exámenes no treponémicos (VDRL o RPR), en dos momentos de la gestación (antes y después de la 20 semanas). Si el examen es positivo, se debe tratar a la mujer y su 
pareja.

Actualmente debido a la cantidad de falsos positivos se eliminó definitivamente el VDRL de sangre de cordón y se determinó realizar exámenes al recién nacido sólo dependiendo del resultado materno en el parto. ${ }^{1,2,3}$

Los principales factores de riesgo han estado asociados a la infección por sífilis materna14: indicadores de nivel socio-económicos, escolares bajos y características de comportamiento individual como consumo de drogas ilegales, consumo de tabaco y alcohol asociados al Virus de Inmunodeficiencia Humana (VIH), prácticas sexuales de alto riesgo la presencia de múltiples parejas sexuales, inicio de la actividad sexual a una edad temprana y defectos en los programas de control prenatal en Infecciones de Transmisión Sexual. La Sífilis Materna y la sífilis congénita los mayores riesgos de sífilis congénita son un limitado número de consultas prenatales y una baja frecuencia en realización de pruebas diagnósticas de sífilis durante dichas consultas pueden tener consecuencias letales, las cuales son prevenibles; sin embargo, aún existen barreras para el acceso a los servicios de control prenatal y la discriminación asociada a las ITS son factores determinantes en el manejo y control de esta enfermedad. ${ }^{1,2,4,5}$.

En un estudio en el Hospital Materno Infantil German Urquidi en la ciudad de Cochabamba, Bolivia, entre marzo y abril de 2003. 489 mujeres estudiadas, 22 resultaron positivas tanto a la prueba de RPR dando una prevalencia de sífilis materna de 4,5\% El objetivo del presente estudio es establecer la prevalencia de Sífilis en mujeres gestantes que acudieron para hacer sus controles prenatales al Hospital de Villa Pagador en la gestión 2015.

\section{METODOLOGÍA.}

Se realizó un estudio retrospectivo descriptivo de corte transversal; recolectando resultados laboratoriales de enero del 01 de 2015 a enero 01 de 2016 ; en el Hospital de Villa Pagador de Cochabamba, Bolivia. El grupo poblacional son mujeres embarazadas que realizaron su control laboratorial antes de las 20 semanas de gestación en el hospital, en una población total de 972 mujeres embarazadas antes de las 20 semanas gestacionales.

La prueba no treponémica que se realizó para la detección de Sífilis fue RPR (reagina plasmática rápida) es una prueba que busca anticuerpos presentes en la sangre es más sensible (casi $100 \%$ ) durante las etapas medias de la sífilis y es menos sensible durante las etapas más tempranas y más tardías de la infección.

\section{RESULTADOS.}

Durante el 2015 se hicieron laboratorio con la prueba de RPR para diagnóstico de sífilis a 972 mujeres embarazadas antes de las 20 semanas de gestación que acudieron al Hospital de Villa Pagador de los cuales 965 resultaron negativos $99 \%$ y solo 7 resultaron positivo 1\%. Ver gráfico 1. 


\section{GRÁFICO \# 1 \\ SÍFILIS EN MUJERES EMBARAZADAS DEL HOSPITAL VILLA PAGADOR}

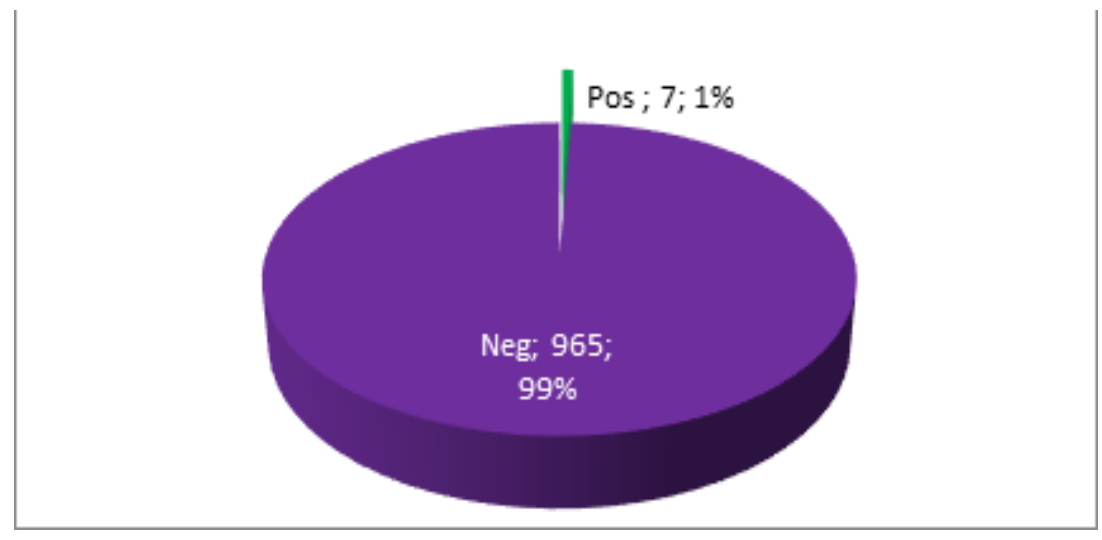

\section{DISCUSIÓN.}

Mediante las pruebas del RPR específicas, se detectó que el $1 \%$ de toda la población en estudio, tiene sífilis materna en el hospital de Villa Pagador el cual es de menor a la prevalencia global de sífilis materna de $4,3 \%$ reportada para Bolivia en 2014. Este resultado apoya la hipótesis de una disminución significativa de sífilis materna entre las mujeres que acuden a los hospitales públicos en Bolivia. Debe seguir con la prevención y limitación del daño en el centro de salud, como en el caso de ser una madre infectada con sífilis, es preciso someterse a una serie de estudios y pruebas clínicas que confirmen la infección. Es importante seguir un control prenatal adecuado, incluyendo estudios serológicos.

A nivel comunitario, es importante llevar a cabo una adecuada educación sexual en las escuelas y centros públicos, así como proveer de servicios de diagnóstico y tratamiento tempranos.

Enseñanza y promoción de métodos de prevención (preservativos, por ejemplo) a través de todos los medios de comunicación.

\section{BIBLIOGRAFÍA.}

1. Organización Panamericana de la Salud. Eliminación de la sífilis congénita en América Latinay el Caribe. Marco de referencia para su implementación. Washington, D.C.: OPS, 2005.

1. Ministerio de Salud: Normas Nacionales de ETS, (1993).

2. Nayrah Villazón-Vargas. Prevalencia de sífilis materna y evaluación de una prueba diagnóstica rápida en Cochabamba, Bolivia.

3. SouTHWicK KL, Blanco S, Santander A, Estenssoro M, Torreo F, Seoane G et al. 
Maternal and congenital syphilis in Bolivia, 1996: prevalence and risk factors. Bull World Health Organ 2001; 79: 33-42.

4. Mandell,Douglas,BennettMandell: Principles and Practice of Infections Diseases (7 ed.). EstadosUnidos: Elsevier. .(2009).

5. Rosen, T., Vandergriff, T., Harting, M.Antibiotic Use in Sexually Transmissible Diseases. DermatolClin 27 (2009) 49-61.

6. Velásquez, H. Manual de enfermedades de transmisión sexual. San José, Costa Rica: EDNASSS-CCSS (1996).

7. Wöhrl, S. Clinical update: syphilis in adults. The Lancet, 369: 1912-1914. (2007). 